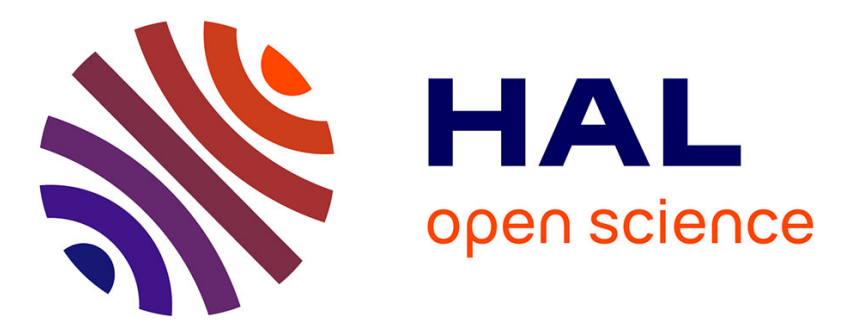

\title{
Muscular responses during motor imagery as a function of muscle contraction types
}

Aymeric Guillot, Florent Lebon, David Rouffet, Stéphane Champely, Julien Doyon, Christian Collet

\section{To cite this version:}

Aymeric Guillot, Florent Lebon, David Rouffet, Stéphane Champely, Julien Doyon, et al.. Muscular responses during motor imagery as a function of muscle contraction types. International Journal of Psychophysiology, 2007, 66 (1), pp.18-27. 10.1016/j.ijpsycho.2007.05.009 . hal-02345780

\author{
HAL Id: hal-02345780 \\ https://hal.science/hal-02345780
}

Submitted on 19 Nov 2021

HAL is a multi-disciplinary open access archive for the deposit and dissemination of scientific research documents, whether they are published or not. The documents may come from teaching and research institutions in France or abroad, or from public or private research centers.
L'archive ouverte pluridisciplinaire HAL, est destinée au dépôt et à la diffusion de documents scientifiques de niveau recherche, publiés ou non, émanant des établissements d'enseignement et de recherche français ou étrangers, des laboratoires publics ou privés.

\section{다)(1) $(5$}

Distributed under a Creative Commons Attribution - NonCommercial| 4.0 International 


\title{
Muscular responses during motor imagery as a function of muscle contraction types
}

\author{
A. Guillot ${ }^{\text {a,* }}$, F. Lebon ${ }^{\text {a }}$, D. Rouffet ${ }^{\text {a }}$, S. Champely ${ }^{\text {a }}$, J. Doyon ${ }^{\text {b }}$, C. Collet $^{\text {a }}$ \\ ${ }^{a}$ Université de Lyon, Université Lyon 1, EA 647, Centre de Recherche et d'Innovation sur le Sport (C.R.I.S.), 27-29 boulevard du 11 Novembre 1918, \\ 69622 Villeurbanne Cedex, France \\ b Functional Neuroimaging Unit, University of Montreal Geriatric Institute, Department of Psychology, University of Montreal, \\ 4565, Queen-Mary Street, Montreal, Quebec, Canada H3W 1W5
}

\begin{abstract}
This study was designed to gain more insight into the mechanisms underlying motor imagery (MI). While there is ample evidence that motor performance and MI share common central neural mechanisms, the question whether MI is accompanied by subliminal electromyographic (EMG) activity remained unsolved. Thirty right-handed volunteers were asked to lift or to imagine lifting a weighted dumbbell using different types of muscle contraction, i.e. heavy concentric, light concentric, isometric and eccentric contractions. EMG activity from 9 muscles of the dominant arm (agonist, antagonist, synergist and fixator muscles) was monitored. Autonomic nervous system responses were also recorded on the non-dominant hand, thus attesting mental activity at the peripheral level. A significant increased pattern of EMG activity was recorded in all muscles during MI, when compared to the rest condition, while the goniometric data did not reveal any movement. Although being subliminal, the magnitude of this activation was found to be correlated to the mental effort required to lift a weight mentally, as more EMG activity was recorded during imaginary lifting of heavy than light concentric contractions. When considering the different types of contraction, our results provided evidence of selective changes in EMG activity. Especially, the imagined eccentric condition elicited a significant weaker muscular activity than all other conditions. In addition, the changes in the EMG pattern mirrored those usually observed during physical movement. These findings support the hypotheses of a selective effect of MI at the level of muscular activity and of incomplete inhibition of the motor command during MI.
\end{abstract}

Keywords: Motor imagery; EMG; Muscle contraction

\section{Introduction}

Motor Imagery (MI) is defined as the mental simulation of a specific action without any corresponding motor output, and shares similar mechanisms underlying movement preparation and execution (Jeannerod, 1994; Decety, 1996). While the effects of MI to improve motor task performance are wellestablished (Feltz and Landers, 1983; Driskell et al., 1994), only a few body of research has been carried out to investigate the effect of MI on strength tasks. Even though Herbert et al. (1998) have challenged the hypothesis that mental training may increase the isometric strength, strong positive effects of MI on voluntary strength of both distal and proximal muscles have

\footnotetext{
* Corresponding author. Tel.: +33 4724316 25; fax: +33 472432846 .

E-mail address: aymeric.guillot@univ-lyon1.fr (A. Guillot).
}

usually been reported (Cornwall et al., 1991; Yue and Cole, 1992; Smith et al., 2003; Zijdewind et al., 2003; Ranganathan et al., 2004; Sidaway and Trzaska, 2005). These results provide evidence of the neural origin of strength gain that occurs before muscle hypertrophy, hence driving the motor units to a higher intensity and/or leading to the recruitment of motor units that remain otherwise inactive. As discussed in the recent paper by Folland and Williams (2007), whilst further research is clearly required, this evidence suggests that substantial increase in the strength of major ambulatory muscle groups can be made without physical activity and be independent of morphological adaptations.

Among the theories supposed to explain such positive effects, the psycho-neuromuscular theory postulates that an electromyographic (EMG) activity of the same muscles occurs during MI and actual movement, although remaining different. 
Such hypothesis has early been proposed by Washburn (1916), who suggested that slight muscle movements were made during MI, and later substantiated by several authors (Jacobson, 1931, 1932; Shaw, 1938), who have reported that this EMG activity occurred only in the muscles that are involved in the movement. In addition, the Golgi tendons organs may be stimulated, and thereby generate neuromuscular feedback (Schmidt and Lee, 1999). This remains a fine hypothesis, as Jami (1992) has demonstrated that the Golgi tendons organs are sensitive to a single motor unit contraction, thus showing the high sensibility of these sensitive structures. Such neuromuscular effects may explain the motor performance improvement. Another hypothesis stated by Jeannerod (1994) is that the residual EMG activity might originate from an incomplete motor command inhibition, MI being in this way considered a tiny muscular contraction (Bonnet et al., 1997).

Therefore, several data have suggested that MI is accompanied by EMG activity and even by specific selective muscle activation (Bird, 1984; Jowdy and Harris, 1990; Gandevia et al., 1997; Hashimoto and Rothwell, 1999). These authors systematically recorded a subliminal activity, the magnitude of the activation being a fraction of that observed during actual performance (for review, see Guillot and Collet, 2005a). The content of the mental image has also been found to be reflected in the magnitude and location of EMG pattern. Internal imagery then results in significantly higher muscles excitation than external imagery (Harris and Robinson, 1986; Bakker et al., 1996). Furthermore, Bakker et al. (1996) and Boschker (2001) have reported that during MI of a movement involving one arm, muscular activity was higher in the active than in the passive arm, and that imagining lifting a heavy object resulted in more EMG activity than that of a lighter object $(9 \mathrm{~kg} v s .4 .5 \mathrm{~kg})$. These data therefore suggest that the physiological responses reflect the spatial movement organization and the quantitative characteristics of MI.

However, the question of recording EMG activity during MI has received inconsistent responses, as muscular quiescence has also been observed during MI (Yue and Cole, 1992; Lotze et al., 1999; Mulder et al., 2004, 2005; Gentili et al., 2006), even when using nuclear magnetic resonance spectroscopy (Decety et al., 1993). Some researchers also required the absence of EMG activity as a precondition to perform a specific MI task (Naito et al., 2002; Jackson et al., 2003). They considered the absence of a significant increase in EMG activity as a proof that the pattern of cerebral activation observed during MI is not due to any movement. Li et al. (2004) have recorded selective muscle activation in the finger flexors in only four of nine subjects during MI, such an observation being later confirmed by Dickstein et al. (2005), during a tip-toe rising task. This latter finding was rather equivocal, as in their last experiment, increased pattern of EMG activity was sometimes, but not systematically recorded during MI, in both healthy subjects and poststroke hemiparetic patients. Furthermore, the EMG activity was also equivocal across all muscles involved during motor performance.

These inconsistent results may originate from methodological problems mainly. First, using surface $v s$. intramuscular EMG recordings might explain why EMG activity may be recorded in some cases but not in the others, although most experiments in this research area used surface EMG. Second, the EMG signal and its properties can be affected by the measurement technique and the experimental conditions, such as electrode geometry, distance between active fibers and electrodes, or location of the muscle (for review, see Kleissen et al., 1998). Finally, differences in the literature may be explained by individual variation in motor command inhibition and could also be related to the experimental conditions (Jeannerod, 1994; Jeannerod and Frak, 1999). Furthermore, the deep muscle fibers are known to fire during the movement preparation mainly (Mellah et al., 1990), and may belong to slow tonic group fibers, thus making their activity difficult to detect by a specific configuration of surface electrodes.

Despite these inconsistent results, the detection of an EMG activity during MI remains an acute field of research. The present study is designed to explore muscular responses during actual lifting a dumbbell and MI of the same movement. Our first aim is to investigate EMG activity of muscles acting as prime movers during actual movement, but also that of antagonist, synergist and fixator muscles, which are simultaneously involved in holding the weight. The second objective is to gain insight into EMG activity characteristics recorded during MI, as a function of muscle contraction types and load constraints (dumbbell weight). Despite the wide range of factors that may affect the EMG signal and inconsistent results described in the previous section, it was expected to record a subliminal EMG activity during MI, in the muscles usually involved during the physical execution of the task. Taken into consideration the EMG activity recorded during actual movement (e.g. Moritani et al., 1988; Fang et al., 2001; McHugh et al., 2002), we further hypothesized that EMG amplitude would differ as a function of the muscle contraction type during MI, i.e. the highest amplitude being recorded during concentric contraction, the lowest amplitude during eccentric contraction, and the "intermediate" amplitude during isometric contraction. In addition, the concentric contraction intensity was also expected to be differentiated through EMG activity during MI, i.e. with a heavy $v s$. a light weight. Finally, among the various techniques used to investigate MI, and according to the aim of the experimental work, the combination of autonomic nervous system (ANS) recording, mental chronometry and well-established psychological tests was considered the most reliable methodological approach to investigate MI directly in the field (for review, see Guillot and Collet, 2005a). Consequently, an ANS response pattern should be recorded after each MI instruction, thus attesting to mental work quality during each trial (Roure et al., 1999; Guillot et al., 2004). Movement duration was expected to be overestimated during MI (Guillot and Collet, 2005b), but MI duration should not be dependent upon muscle contraction type.

\section{Methods}

\subsection{Participants}

Thirty right-handed sport students (15 men and 15 women, aged from 18 to 25 , mean $=21.2 \pm 2.4$ ) gave their informed 
consent to take part in the experimental procedures, which were approved by the local research ethics board. By self-report, none had any neuromuscular disorder, and all were free of any recent muscular injury. They were not made aware of the purposes or hypotheses of the experiment until after test completion.

\subsection{Experimental procedures}

During a preliminary session, planned 7 days before the experiment, all participants performed an incremental test to determine their maximum lifting performance (1RM) when lifting a weighted dumbbell using a bending movement of the forearm on the arm. The best mark resulting from one repetition (concentric contraction) was considered the 1RM. The isometric maximal voluntary force (IMVC) was also measured using a strain gauge force transducer with the elbow joint positioned at $90^{\circ}$. Finally, participants completed the Revised version of the Movement Imagery Questionnaire (MIQ-R, Hall and Martin, 1997 ) in a quiet room. The MIQ-R is made up of 8 items known to evaluate individual differences in visual (4 items) and kinesthetic (4 items) movement imagery. Completing each item requires 4 steps. First, the starting position is described. Second, a specific arm, leg or whole body movement is precisely described and then, the participant is required to perform it. Third, each individual is asked to reassume the starting position and to imagine the movement, using visual or kinesthetic imagery alternatively (no actual movement is made). Finally, a score is assigned using a 7-point scale regarding the ease/difficulty associated with representing each movement mentally.

During the experimental procedure, participants were seated comfortably in an experimental chair after a specific warm-up including incremental dumbbell lifting exercises. To avoid any compensatory movement, participants were fixed against the chair (Fig. 1). The experimenter then attached the EMG and ANS electrodes, on the active arm and the non-dominant hand respectively. A goniometer (SG110, Biometrics) was also strapped to the active arm and forearm, to control the initial position and check that the elbow angle $\left(90^{\circ}\right)$ remained constant during MI (Fig. 1), thus attesting that the participant did not move. The participants were then asked to lift or to imagine

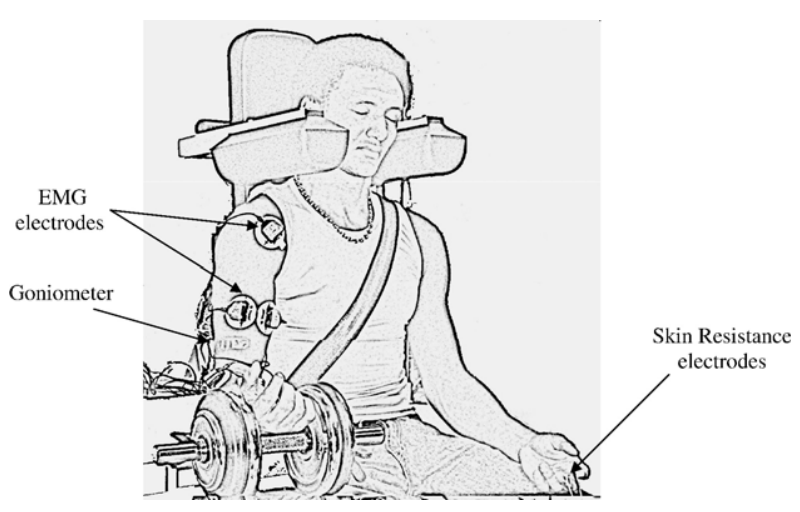

Fig. 1. Measurement equipment and subject positioning. The part of the goniometric system located in the forearm is not visible in this drawing. lifting the weighted dumbbell. The four conditions were i) heavy concentric contraction ( $80 \%$ of $1 \mathrm{RM})$, light concentric contraction $(50 \%$ of $1 \mathrm{RM})$, iii) isometric contraction (at least $95 \%$ of the maximal isometric strength) with the strain gauge force transducer, and iv) eccentric contraction (120\% of 1RM). A total of 8 experimental conditions were therefore counterbalanced for each contraction type. Four actual and 8 imagined trials were performed for each experimental condition, i.e. a total of 16 actual movements and $32 \mathrm{MI}$ trials. As each trial was preceded by a rest-period, EMG and ANS activities were also recorded during a total of 32 rest-periods, to be compared with those recorded during actual performance and MI.

During actual performance, participants were asked to lift the dumbbell as fast as possible (concentric contractions), to "lower" it as much as possible (eccentric condition), or to hold the isometric contraction during a 5s-period. During MI, they were instructed to imagine the movement as if they were actually performing it, using an association of internal visual and kinesthetic imagery. Specifically, this latter component appears essential, as highlighted by Neuper et al. (2005). Each trial was separated from the next by a rest period, which was never less than 20s, thus allowing ANS variables to recover basal levels and EMG recordings to keep stable baseline. Upon mental initiation of the first body movement and at the end of the sequence, athletes pressed a button with their non-dominant hand, to start and stop the timer. A MI script was developed to standardize the instructions. Each participant was read this script and therefore received the following instructions before the first MI trial: "Try to imagine yourself doing the motor sequence with your eyes closed by imagining the different movements, as if you had a camera on your head, and perceiving the body sensations. You see and feel only what you would if you actually performed this particular skill. Start the timer with your non-dominant hand, as soon as you have left the reference point (initial position), which means at the beginning of the first imagined movement. Imagine the entire sequence and stop the timer at the end of the contraction. Make sure you use imagery in the way that is most comfortable to you, but without moving your arm". To check that they performed exactly the correct type of imagery, athletes were regularly asked to describe the nature of the images after MI. During this debriefing, athletes were also instructed to describe the difficulty they encountered to imagine the movement and then, to rate a score of MI quality, using a 4points scale. This was set as follow: $1=$ very easy to imagine/feel and $4=$ very difficult to imagine/feel ( 2 and 3 being intermediate levels).

\subsection{Mechanical recordings}

Imagined and actual durations were recorded by the experimenter, during each muscle contraction type, mean actual duration being considered the reference. In the concentric and eccentric muscle contraction conditions, the actual durations were calculated from the signal recorded, while using a goniometer (SG110, Biometrics) strapped to the active arm and forearm. In the isometric contraction condition, the subjects were asked to maintain over $90 \%$ of their IMVC (recorded 
during the preliminary session) during $5 \mathrm{~s}$ with the elbow joint positioned at $90^{\circ}$. The angle of the elbow joint and the magnitude of the force applied were controlled using the goniometer and the strain gauge force transducer, respectively. In the imagined contraction conditions, the goniometer was used to attest the absence of any elbow movement during MI.

\subsection{Electrical recordings}

Before placing the EMG surface electrodes, the skin was shaved, abraded and cleaned with alcohol swabs to improve the contact between the skin and the electrodes but also to reduce skin impedance. Raw EMG signals were simultaneously recorded with the Flex-comp Infinity system (Thought technology, Montreal, Canada), from the long head of the biceps brachii (LBB), short head of the biceps brachii (SBB), brachioradialis (BR), triceps brachii (TB), flexor carpi ulnaris (FCU), flexor carpi radialis (FCR), anterior deltoid (DEL), superior trapezius (TRA) and major pectoralis (PEC) muscles. Raw EMG signals were sampled at $2048 \mathrm{~Hz}$ on a computer before being further processed. Then the root mean squared value of the EMG signals (EMGrms) was calculated using a time averaging period of $25 \mathrm{~ms}$. The mean EMGrms values of the nine muscles were calculated over the imagined and actual durations of the different contraction types.

Autonomic nervous system recordings were aimed at evaluating MI quality through more objective data than selfevaluation (Roure et al., 1999). High brain functioning may be studied through ANS effector activity at a peripheral level (Hugdahl, 1996) which anticipate and accompany behavior, thus representing non-conscious physiological mechanisms of central mental processes. Cutaneous autonomic effectors, e.g. sweat glands are innervated by sympathetic endings only, their activation eliciting sweat release and, consequently, a decrease in skin resistance. Several experiment demonstrated that MI quality may be estimated by ANS responses, especially through skin resistance (Roure et al., 1999; Guillot et al., 2004; Guillot and Collet, 2005a). Instrumentation was designed by the Biomedical Microsensors and Microsystems Laboratory of the French National Institute of Applied Sciences (Dittmar et al., 1995). Signal sampling was carried out at a frequency of $10 \mathrm{~Hz}$. Skin resistance was recorded using two $30 \mathrm{~mm}^{2}$ unpolarizable $\mathrm{Ag} / \mathrm{AgCl}$ electrodes (Clark Electromedical Instruments, Ref. E243) placed on the second phalanx of the second and third digits of the non-dominant hand, and held by adhesive tape (Fowles et al., 1981). Skin resistance was measured with $15 \mu \mathrm{A}$ current (current density $=0.5 \mu \mathrm{A} / \mathrm{mm}^{2}$ ), and an isotonic conductive paste (TECA ref: 822-201210) was used to improve skin/electrode contact As response amplitude depends on the pre-stimulation value (Furedy and Scher, 1989), a more reliable index was defined without referring to the initial value (tonic level). The Ohmic Perturbation Duration (OPD) is measured at the beginning of the sudden drop elicited by MI, and ends when the slope, while recovering basal level, shows no more fluctuations, and when the slope is similar to that observed before the stimulation (Vernet-Maury et al., 1995).

\subsection{Data analysis}

The first step of statistical EMG analysis was to test whether a difference may be observed between males and females by using a Multivariate analysis of variance (MANOVA). As the male/female comparisons did not reach the $5 \%$ threshold, male and female data were pooled and the variable related to sex was no more taken into account. A standard MANOVA test was thus performed for the 8 variables ( 2 movement conditions, i.e. actual performance and MI and 4 types of muscles contraction as previously described). All statistical computations were made by using a transformation $(1 / y)$ to fit the usual normal distribution assumptions. To explore the structure of the variables, these were re-expressed using a contrast $\mathrm{H}$ matrix in terms of derived variables leading to better distinguish the main single effects (movement conditions and types of contraction) from their interaction (conditions * types of contraction). A suitable $\mathrm{H}$ matrix produced a first derived variable (not of interest here) giving the overall level of the responses. A second derived variable summarized the main effect of conditions, while 3 derived variables $(3,4$ and 5$)$ measured the main effect of the types of contraction, the 3 last ones (6,7 and 8) summarizing the interaction effect (incidentally, this $\mathrm{H}$ matrix was exactly the design and analysis of the example 2.2 in the chapter about MANOVA of Hand et al., 1996). The nullity of the interaction was first tested by using the Hotelling's T2-test on the 3 last derived variables. Then, we examined the main effect of types of contraction by the same multivariate Hotelling's test applied to the derived variables 3, 4 and 5. In a first part, only the long biceps was considered as this is the main muscle involved in the elbow flexion. In a second part, the other muscles are considered, using the same analysis.

MIQ-R scores, ANS responses (OPD), and both physical execution and MI durations comparisons were made using Student's paired t-tests. The results are presented as mean (standard deviation values), the alpha level being set at $p<0.05$.

\section{Results}

\subsection{Behavioral results}

The mean MIQ-R score was 43.97 (5.9). No difference was found between males and females $(t=0.641, p>0.05$, NS), mean scores being 43.27 (5.91) and 44.67 (6.04) respectively. When considering the visual and kinaesthetic subscales, the mean scores were 23.94 (3.5) and 19.87 (3.8), respectively. During the debriefing following the MI sessions, participants reported that they used the MI outlined in the script, without any switch between external and internal MI. None reported changing the imagery script to suit individual needs, but rehearsed the motor sequence as they were requested to do. The evaluation of self-reported MI accuracy did not show any difference among the experimental conditions $(F=2.92$, $p>0.05$, NS). Similarly, these scores did not change across trials. Mean values (SD) for the 4-points scale for heavy concentric, light concentric, isometric and eccentric contraction 
conditions were $2.6(0.7), 2.5(0.7), 2.9(0.7)$ and $2.8(0.8)$, respectively.

The average time difference between the actual and the imagined trials was compared for each type of muscle contraction. Mean durations of actual concentric contractions were $1.21 \mathrm{~s}(0.2)$ and $0.78 \mathrm{~s}(1.1)$ for the heavy and light weights respectively. Imagined concentric contraction times were longer than actual times ( $2.38 \mathrm{~s}(0.1)$ and $1.62 \mathrm{~s}(1.1)$ respectively). The comparisons between imagined and physical times reached significance $(t=-5.5, p<0.001$ and $t=-3.96, p<0.001)$. Under the $5 \mathrm{~s}$ isometric condition, actual and MI times did not differ $(t=0.3, p>0.05$, NS), mean MI duration being $4.89 \mathrm{~s}$ (2). Similarly, in the eccentric condition, MI times did not differ from physical execution times $(t=0.5, p>0.05$, NS), mean durations being $4.25 \mathrm{~s}(2.2)$ and $4.02 \mathrm{~s}$ (1.9) respectively. Interestingly, there was no significant gender difference, whatever the muscle contraction condition.

The goniometer measured the elbow angles during both the rest and the MI conditions with the aim to check whether during MI, subjects were able to keep the precise angular position that they were requested to (elbow angle of $90^{\circ}$ ), and thus to prevent any movement during imagined contractions. No significant difference was found ( $t=1.39, p>0.05$, NS), average values being $0.52^{\circ}(0.2)$ and $0.55^{\circ}(0.2)$ during the MI and rest conditions respectively.

\subsection{EMG activity during $M I$}

All statistical computations were made by using normalized data, i.e. $1 / \mathrm{y}$. When considering the EMG activity of the long head of the biceps brachii, the main muscle involved in the elbow flexion, the gender comparison did not reach the significant threshold $(F(8,28)=2.11$, [Pillai $=0.45], p>0.05)$. Hence, males and females data were pooled. Similarly, none of the EMG activity provided differences between males and females for the 8 other muscles. All p-values were upper than the significant threshold. The forthcoming statistical test then used the Hotelling T2. The aim was to create derivate variables to separate interactions from simple effects $(2$ movement condi-

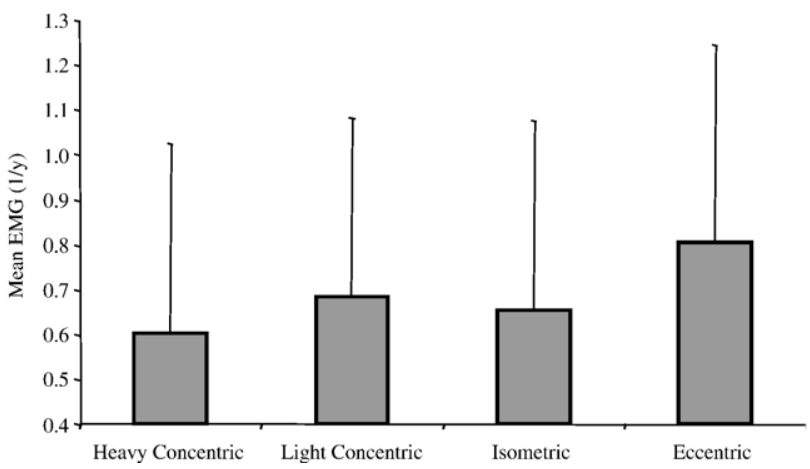

Fig. 2. EMG activity of the long head of the biceps brachii $(1 / y)$ as a function of the type of muscle contraction. The eccentric contraction elicited the weakest EMG activity whereas the heavy concentric contraction elicited the highest. Further, there was a significant difference between the MI of the heavy concentric muscular contraction compared with the light concentric muscular contraction.
Table 1

Mean EMG activity recorded during the rest and motor imagery conditions

\begin{tabular}{|c|c|c|c|}
\hline & $\begin{array}{l}\text { Mean rest EMG } \\
\text { activity }(1 / y)\end{array}$ & $\begin{array}{l}\text { Mean MI EMG } \\
\text { activity }(1 / y)\end{array}$ & $P$-value \\
\hline $\begin{array}{l}\text { Biceps brachii } \\
\text { (long head) }\end{array}$ & 0.82 & 0.69 & $<0.001$ \\
\hline $\begin{array}{r}\text { Biceps brachii } \\
\text { (short head) }\end{array}$ & 0.79 & 0.66 & $<0.000$ \\
\hline Anterior deltoidus & 0.66 & 0.59 & $<0.000$ \\
\hline Superior trapezius & 0.28 & 0.26 & $<0.01$ \\
\hline Triceps brachii & 0.86 & 0.78 & $<0.01$ \\
\hline Brachioradialis & 0.68 & 0.51 & $<0.01$ \\
\hline Flexor carpi ulnaris & 0.83 & 0.73 & $<0.02$ \\
\hline $\begin{array}{l}\text { Flexor carpi } \\
\text { radialis }\end{array}$ & 0.73 & 0.65 & $<0.01$ \\
\hline Major pectoralis & 0.35 & 0.32 & $<0.002$ \\
\hline
\end{tabular}

Results clearly indicated higher, although subliminal, muscular activity during MI than during rest.

tions [MI and rest condition] * 4 types of muscle contractions). The matrix of transformations $\mathrm{H}$ yielded to 8 derivate variables from the 8 first variables. The 3 last variables were aimed to test interaction. With reference to the matrix, it was verified whether the 3 variables showed a mean different from $(0,0,0)$ : $\mathrm{T} 2=8.1$, $p=0.08$. As the critical probability was $8 \%$, we considered that no interaction occurred among the 3 variables. Thus, the effect of movement conditions on the one hand, and type of muscle contraction on the other, were tested as separate variables.

The Hotelling T2 test showed a marginally effect of the muscle contraction type condition for the long head of the biceps brachii, as shown by Fig. 2 (T2 $=8.23, p=0.07$ ). However, the light concentric contraction condition elicited less muscular activity than the heavy concentric contraction. In addition, the eccentric condition elicited the significant weakest muscular activity (Fig. 2).

When considering EMG recordings of the 8 remaining muscles, none of the $8 p$-values reached the significant threshold, although few of them indicated a marginally significant $p$-value (flexor carpi ulnaris, $p=0.09$ and major pectoralis, $p=0.06$ ). Consequently, the effect of each condition was investigated. A significant effect of the contraction type was found in the anterior deltoidus $(p=0.03)$, brachioradialis $(p=0.01)$, flexor carpi ulnaris $(p=0.002)$, and flexor carpi radialis levels $(p<0.001)$. In addition, the Hotteling $\mathrm{T} 2$ test provided a marginally significant difference in the major pectoralis $(p=0.08)$. The weakest muscle activity was provided during eccentric MI of lifting the weight. There was also a strong difference between the two concentric conditions, heavy concentric contraction eliciting higher muscular activity than light concentric contraction. At last, no clear difference emerged from the comparison of heavy concentric and isometric contractions.

\subsection{EMG activity during $M I v s$. rest}

Again, all statistical computations were made by using normalized data, i.e. $1 / y$. The statistical analysis revealed a greater EMG activity recorded during MI than during the rest condition. Such difference was also observed in the other 8 muscles, as 
shown by Table 1 . To emphasize data reliability, it was checked whether participants remained motionless during the MI sequences (at least that they did not show more muscular activity than during the rest control condition). Thus, goniometric data were compared to check that there was no significant difference between the rest and the MI conditions.

Greater EMG activity were recorded in the long head of the biceps brachii during MI than during the rest condition, average normalized values being $0.82 \mathrm{mV}(0.4)$ and $0.69 \mathrm{mV}(0.4)$ respectively. It is noticeable that the $4 \mathrm{MI}$ conditions were considered in this comparison. Similar results were obtained in each muscle recording (Fig. 3). When analyzing each type of muscle contraction in a separate way, similar results were observed. The EMG activity of the long head of the biceps was significantly greater during $\mathrm{MI}$ than during the rest condition the heavy concentric ( $\mathrm{t}=2.8, p<0.01)$, the light concentric $(t=2.4$, $p<0.02)$, the isometric $(t=2.5, p=0.016)$ and the eccentric conditions $(t=2.6, p=0.014)$. These statistical differences were also obtained when analyzing the data of all muscles, with the exception of the short head of the biceps brachii and triceps brachii during the eccentric condition, the flexor carpi radialis during the isometric condition, and the trapezius, flexor carpi ulnaris and radialis during the heavy concentric condition.

\subsection{EMG activity during physical execution}

A significant difference was found between males and females EMG in the long head of the biceps brachii (Pillai $=0.565$, $F(4,18)=5.85, p<0.01)$. Mean males EMG values were $1001 \mathrm{mV}, 832 \mathrm{mV}, 862 \mathrm{mV}$, and $696 \mathrm{mV}$ in the isometric, heavy concentric, eccentric and light concentric conditions respectively. Weaker EMG values were recorded in women (479 mV, $478 \mathrm{mV}, 421 \mathrm{mV}$, and $332 \mathrm{mV}$, respectively). Comparing each type of contraction to each other provided evidence of a systematic effect of the type of contraction, showing that the EMG activity recorded during the light concentric contraction was weaker than those recorded during other types of contraction (Table 2). Further, in women, a tendency was also observed when
Table 2

Comparison of the EMG activity as a function of muscle contraction type

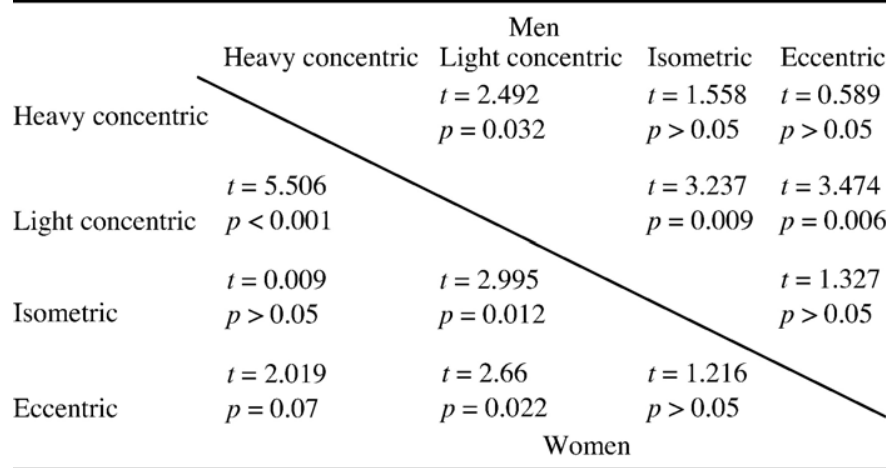

A systematic effect of muscle activity was observed in a way that the EMG activity recorded during both the light concentric and the eccentric contraction was significantly weaker than that recorded during other types of contraction both in men and women.

comparing the EMG activity recorded during the heavy concentric and the eccentric conditions $(t=2.02, p=0.07)$.

When considering the other 8 muscles, different patterns of EMG activity were recorded, so that no clear conclusion may be drawn. In the male group, for example, greater EMG values were recorded during the isometric condition when compared to other types of contraction, except in the short head of the biceps brachii and the deltoïdus activity (greater EMG activity during the eccentric than during the isometric condition), as in the trapezius and the deltoïdus activity (greater activity during the heavy concentric than during the isometric condition). Similarly, the weakest EMG activity was recorded during the light concentric contraction, with the exception of the brachioradialis activity (greater activity during the eccentric than during the light concentric condition). Finally, no significant difference was observed between the heavy concentric and the eccentric conditions, except in the activity of the flexor carpi ulnaris. In the female group, no significant difference was recorded between the isometric condition and the other types of contraction, as between the eccentric and the heavy concentric

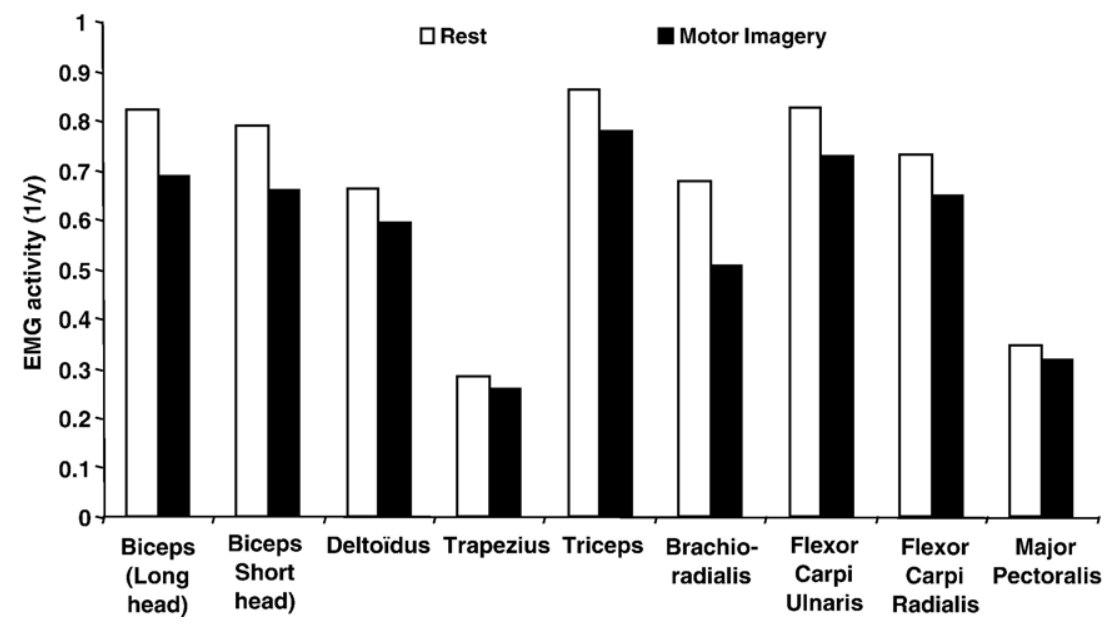

Fig. 3. EMG activity $(1 / y)$ during MI and rest conditions. The MI condition was shown to elicit a higher EMG activity than that recorded during the rest condition in all muscles. 
Table 3

Ohmic perturbation duration recorded during physical execution and motor imagery

\begin{tabular}{lllllll}
\hline Contraction type & Condition & OPD (s) & Standard deviation & $t$ & $p$ \\
\hline Heavy concentric & Actual & 5.26 & 2.1 & 7.14 & $<0.001$ \\
& MI & 2.13 & 1.11 & & \\
Light concentric & Actual & 4.86 & 2.38 & 5.98 & $<0.001$ \\
& MI & 1.48 & 1.2 & & \\
Isometric & Actual & 7.91 & 2.38 & 11.4 & $<0.001$ \\
& MI & 2.21 & 1.33 & & \\
Eccentric & Actual & 9.04 & 2.38 & 12.7 & $<0.001$ \\
& MI & 1.26 & 0.89 & & \\
\hline
\end{tabular}

ANS responses recorded during MI were found to be significantly shorter than those recorded during physical execution.

conditions. By contrast, the weakest EMG activity was recorded during the light concentric condition, with the exception of the brachioradialis, the short head of the biceps brachii and the trapezius, when compared to the eccentric condition.

\subsection{ANS activity}

Skin resistance responses (measured through the OPD index) were recorded during both physical and imagined contractions. During MI, the responses were significantly shorter than those recorded during actual practice in all muscle contraction conditions $(p<0.001)$, as shown by Table 3 . As MI quality was estimated through ANS responses, especially skin resistance responses (Guillot et al., 2004; Guillot and Collet, 2005a; Roure et al., 1999), good and poor MI trials were differentiated with respect to the OPD of each MI trial. However, the EMG activity did not statistically differ between these "correct and incorrect" MI trials, with the exception of the long head of the biceps brachii during light concentric contraction $(t=2.39, p<0.05)$ and the brachoradialis during heavy concentric contraction $(t=3.5$, $p<0.001)$. In these muscles, the mean EMG activity was significantly greater during correct than during incorrect MI trials (4.31 (4.2) and 2.97 (3.1) in the biceps brachii activity, 3.55 (1.8) and 2.74 (1.6) in the brachioradialis activity). Finally, the EMG activity tended to be significantly greater during correct heavy concentric than during incorrect MI trials in the long head of the biceps brachii activity $(t=1.77, p=0.09)$, mean values being 4.88 (4.6) and 3.53 (3.12) respectively.

\section{Discussion}

The present study was designed to investigate the muscular responses during the actual lifting of a dumbbell and the MI of the same movement. Two main independent variables were considered, the type of muscle contraction and the weight to lift. The main result was that MI was accompanied by a subliminal specific EMG activity of muscles acting as prime movers during actual movement, but also that of antagonist, synergist and fixator muscles. Further, the statistical analysis revealed a greater EMG activity during MI than during the rest condition, whatever the gender, the muscles and the type of contraction. In addition, there was an effect of the weight being mentally lifted under the concentric condition, and the eccentric condition was found to elicit a significant weakest muscular activity than during all other conditions, hence suggesting that the low magnitude of the physiological response may interact with the type of contraction being imagined. These results provide further evidence that MI and motor execution share common neural mechanisms. This was confirmed through mental chronometry as no difference emerged from actual and imagined duration, perhaps with the exception of the concentric conditions. This latter difference is easily explained with reference to the very short actual duration (less than $1.5 \mathrm{~s}$ ), as rapid movement duration is always overestimated during MI (Guillot and Collet, 2005b).

First, our results support the previous findings by Bakker et al. (1996) and Boschker (2001) who found that the MI of lifting $9 \mathrm{~kg}$ weights resulted in a larger EMG activity in the active arm than the MI of lifting $4 \mathrm{~kg} 1 / 2$. In the present study, the MI of a heavy concentric contraction ( $80 \%$ of $1 \mathrm{RM})$ resulted in a greater pattern of EMG activity than during MI of a light concentric condition ( $50 \%$ of $1 \mathrm{RM})$. Especially, the association of visual internal and kinesthetic imagery agrees with the assumptions stating that a larger EMG activity is recorded when subjects use internal rather than external visual imagery (Hale, 1982; Harris and Robinson, 1986). Although some authors have reported that the EMG activity was necessarily equivocal across all muscles that contract during actual performance (Dickstein et al., 2005), we recorded an overall activation in the 9 muscles being monitored. These results corroborates the view stating that the content of the mental image (i.e. the intensity of the contraction) may be related to the magnitude of the subliminal EMG activity, which is thought to partially reflect, in turn, the quantitative characteristics of this mental image. As outlined by Boschker (2001), however, it still remains an empirical question to find out how detailed the EMG response mirrors the image. Especially, such a difference during MI may also be linked to the relationship between physical effort and perceived exertion arising from the tacit knowledge of how difficult is the heavy contraction by comparison to the light condition. Altogether, such a modification in the EMG activity during MI seems more than an epiphenomenon, even though it remains difficult to explain. According to Jeannerod (1994) and Bonnet et al. (1997), this physiological response is supposed to result from an incomplete inhibition of the motor command.

Second, our results provide evidence, for the first time, of an effect of the contraction type. The EMG activity was found larger during the heavy concentric contraction than during both the light concentric and eccentric conditions. More, the pattern of EMG activity recorded during imagined eccentric contractions was systematically weaker than those observed during all other types of contraction. Interestingly, these results are in keeping with previous literature findings showing that the EMG activity recorded during actual eccentric contractions is weaker than that observed during concentric and isometric contractions (Moritani et al., 1988; Tesch et al., 1990; Fang et al., 2001; McHugh et al., 2002). This difference may be explained by a fewer activation of the motor units during eccentric than during concentric contractions at the same force, more fast-twitch motor units being however activated during actual eccentric contractions. Even though a spectral analysis should confirm 
this hypothesis, a similar physiological mechanism may occur during MI. In contrast, while previous studies investigating physical execution have shown that a greater EMG activity was recorded during concentric than during isometric or during eccentric contractions (Komi et al., 2000; Linnamo et al., 2006), we found that the EMG activity was greater during actual isometric than during heavy concentric contractions. This difference may result in the difference between the designs of the two studies: participants were requested to lift $80 \%$ of $1 \mathrm{RM}$ in our experiment while previous studies investigated maximal voluntary contractions. Finally, our results support the findings by Komi et al. (2000) and Linnamo et al. (2006) with regards to MI, as the pattern of activity during imagined contractions was greater during concentric than during isometric conditions. The sensation of effort, which has usually been reported during MI (Decety and Boisson, 1990; Decety and Lindgren, 1991), may explain these results, especially when subjects have to imagine lifting a heavy weighted dumbbell.

The outcomes also indicated that the pattern of EMG activity was recorded in all muscles involved during the physical flexion of the forearm, i.e. the agonist muscles but also the antagonists, synergists and muscles with a fixation function. Agonists create the range of movement in a joint by contracting while the antagonists act in opposition about the axis of the joint. The antagonists which show eccentric activity have often been found activated during MI (e.g. Bakker et al., 1996; Boschker, 2001; Slade et al., 2002). Interestingly, the synergists and the muscles with a fixation function, eliciting single muscle action to reinforce the agonist activity and concerted activation of several muscles respectively, were also activated during MI, in our experiment. This result emphasizes the functional equivalence between MI and motor performance, which has already been well-established with regards to the central and peripheral functional equivalence (e.g. Decety et al., 1994; Pfurtscheller and Neuper, 1997; Holmes and Collins, 2001; Guillot and Collet, 2005a; Guillot et al., submitted for publication), as the behavioral (temporal) equivalence (Guillot and Collet, 2005b). Even though earlier studies found a functional equivalence within the EMG activity (Jacobson, 1931; Shaw, 1940), none has reported an activation of all muscles involved in the production of the actual movement.

The EMG activity recorded during the MI of lifting weighted dumbbells was shown to mirror actual execution, and to distinguish among the different types of contraction elicited by the movement under actual conditions of execution. This lends support that this activity originates from the high central nervous system. Moreover, as EMG activity has been sometimes, but not systematically, recorded during MI (Dickstein et al., 2005), it seems not reasonable to explain the effects of MI on motor performance and learning in terms of bottom-up mechanisms only (Mulder et al., 2004). These authors thus argued that it was more plausible to explain such effects in terms of a top-down mechanism based on the activation of a central representation of the movement (Mulder et al., 2005). They also interpreted that the occurrence of peripheral activity in other studies could not be satisfactorily explained by such a central theory. However, central operations (planning and program- ming) are paralleled by peripheral responses such as ANS responses which anticipate and accompany behavior, thus representing non-conscious physiological mechanisms of central mental processes (Collet et al., 1999; Plascencia-Alvarez et al., 2002; Sequeira and Ba M'hamed, 1999). Therefore, these physiological responses appear compatible with a central origin of MI effects. More, the occurrence of a pattern of EMG activity during mental practice has been interpreted as an incomplete inhibition of the motor output (Jeannerod, 1994; Bonnet et al., 1997). Especially, the cerebellum is thought to be involved in the inhibition of movement execution during MI, the inhibitory process being possibly controlled by the Larsell lobule HVII ipsilateral to the movement (Lotze et al., 1999). Furthermore, the experimental data providing evidence of substantial increase in muscle strength through MI, as mentioned in the introduction, lend also support to the central explanation theory of MI. These results were explained with reference to the neural origin of strength gain, hence driving the motor units to a higher intensity and/or leading to the recruitment of motor units that are otherwise inactive, without any peripheral changes such as hypertrophy. Similar neural reorganizations are supposed to explain the limit of strength loss during immobilization (Newsom et al., 2003). It may thus be concluded that the effect of MI may be explained by the cerebral and cerebellar plasticity following mental practice (Lafleur et al., 2002; Lacourse et al., 2004), hence resulting from a central origin.

Finally, no clear difference was found when comparing the EMG activity recorded during correct and incorrect trials, i.e. when MI was considered, or not, being vivid, with the exception of the agonist muscles (the long biceps brachii in our experiment). Although there was the same tendency in some other muscles towards a larger pattern of EMG during correct trials, the present results do not support the hypothesis stating that subjects who produce more specific patterns of muscular activity are better imagers than those who produce less specific patterns of muscular activity. This finding thus agrees with previous literature data that failed to find a relationship between MI ability and EMG activity (Hale, 1982; Hecker and Kazcor, 1988; Jowdy and Harris, 1990; Boschker, 2001), with the exception of the paper by Lutz (2003). Conversely, this is probably not the case when central nervous system activity is considered (Guillot et al., submitted for publication). Consequently, the recording of EMG activity may be considered a reliable indicator of $\mathrm{MI}$ accuracy with reference to the EMG activity of agonist muscles only (Guillot and Collet, 2005a).

To conclude, the present study shows that a pattern of EMG activity was recorded during MI in all muscles interested in the movement we considered, as a function of the weight to be lifted and to the muscle contraction type. This pattern of muscular response was found to overall mirror the configuration of the EMG activity recorded during different types of physical contraction, even though the magnitude was strongly weaker during MI, as expected. Such equivalence still needs, however, further experimental investigation, using spectral analysis, for example to check whether the configuration of activation of the motor units is similar during MI and motor execution. Altogether, these data lend support to the high central nervous 
system origin of that activity, hence suggesting that the effect of MI may be explained by the central nervous system plasticity following mental practice.

\section{References}

Bakker, F.C., Boschker, M.S.J., Chung, T., 1996. Changes in muscular activity while imagining weight-lifting using stimulus or response propositions. J. Sport Exerc. Psychol. 18, 313-324.

Bird, E.I., 1984. EMG quantification of mental rehearsal. Percept. Mot. Skills 59, 899-906.

Bonnet, M., Decety, J., Jeannerod, M., Requin, J., 1997. Mental simulation of an action modulates the excitability of spinal reflex pathways in man. Cogn. Brain Res. 5, 221-228.

Boschker, M.S., 2001. Action-based imagery: on the nature of mentally imagined motor actions. Doctoral Thesis Dissertation, University of Amsterdam.

Collet, C., Dittmar, A., Vernet-Maury, E., 1999. Programming or inhibiting action: autonomic nervous system control of anticipation. Int. J. Psychophysiol. 32, 261-276.

Cornwall, M.W., Buscato, M.P., Barry, S., 1991. Effect of mental practice on isometric muscular strength. J. Orthop. Sports Phys. Ther. 13, 231-234.

Decety, J., 1996. Do imagined and executed actions share the same neural substrate? Cogn. Brain Res. 3, 87-93.

Decety, J., Boisson, D., 1990. Effect of brain and spinal cord injuries on motor imagery. Eur. Arch. Psych. Clin. Neurosci. 240, 39-43.

Decety, J., Lindgren, M., 1991. Sensation of effort and duration of mentally executed actions. Scand. J. Psychol. 32, 97-104.

Decety, J., Jeannnerod, M., Durozard, D., Baverel, G., 1993. Central activation of autonomic effectors during mental simulation of motor actions in man. J. Physiol. (Lond.) 461, 549-563.

Decety, J., Perani, D., Jeannerod, M., Bettinardi, V., Tadary, B., Woods, R.P., Maziota, J.C., Fazio, F., 1994. Mapping motor representation with positron emission tomography. Nature 371, 600-602.

Dickstein, R., Gazit-Grunwald, M., Plax, M., Dunsky, A., Marcovitz, E., 2005. EMG activity in selected target muscles during imagery rising on tiptoes in healthy adults and poststroke hemiparetic patients. J. Mot. Behav. 37, 475-483.

Dittmar, A., Rada, H., Delhomme, G., Vernet-Maury, E., Collet, C., Roure, R., Unterreiner, R., Robini, M., Delemer, C., 1995. A multi-sensor system for the non-invasive measurement of the activity of the autonomic nervous system. Sens. Actuators, B, Chem. 26-27, 461-464.

Driskell, J.E., Copper, C., Moran, A., 1994. Does mental practice enhance performance? J. Appl. Sport Psychol. 79, 481-491.

Fang, Y., Siemionow, V., Sahgal, V., Xiong, F., Yue, G.H., 2001. Greater movement-related cortical potential during human eccentric versus concentric muscle contractions. J. Neurophysiol. 86, 1764-1772.

Feltz, D.L., Landers, D.M., 1983. The effects of mental practice on motor skill learning and performance: a meta-analysis. J. Sport Psychol. 5, 25-57.

Folland, J.P., Williams, A.G., 2007. The adaptations to strength training. Morphological and neurological contributions to increased strength. Sports Med. 37, 145-168.

Fowles, D.C., Christie, M.J., Edelberg, R., 1981. Publication recommendations for electrodermal measurements. Psychophysiology 18, 232-239.

Furedy, J.J., Scher, H., 1989. The law of initial values: differentiated testing as an empirical generalization versus enshrinement as a methodological rule. Psychophysiology 26, 120-122.

Gandevia, S.C., Wilson, L.R., Inglis, J.T., Burke, D., 1997. Mental rehearsal of motor tasks recruits a-motoneurones but fails to recruit human fusimotor neurones selectively. J. Physiol. 505, 259-266.

Gentili, R., Papaxanthis, C., Pozzo, T., 2006. Improvement and generalization of arm motor performance through imagery practice. Neuroscience 137, 761-772.

Guillot, A., Collet, C., 2005a. Contribution from neurophysiological and psychological methods to the study of motor imagery. Brains Res. Rev. 50, 387-397.

Guillot, A., Collet, C., 2005b. Duration of mentally simulated movement: a review. J. Mot. Behav. 37, 10-20.
Guillot, A., Collet, C., Dittmar, A., 2004. Relationship between visual vs kinaesthetic imagery, field dependence-independence and complex motor skills. J. Psychophysiol. 18, 190-198.

Guillot, A., Collet, C., Nguyen, V.A., Malouin, F., Richards, C., Doyon, J., submitted for publication. Brain activity during visual vs. kinesthetic imagery: an fMRI study. Hum. Brain Mapp.

Hale, B.D., 1982. The effects of internal and external imagery on muscular and ocular concomitants. J. Sport Psychol. 4, 379-387.

Hall, C.R., Martin, K.A., 1997. Measuring movement imagery abilities: a revision of the movement imagery questionnaire. J. Ment. Imag. 21, 143-154.

Hand, D., Crowder, M.J., Hand, H.J., 1996. Practical Longitudinal Data Analysis, 1st edition. Crc Press Llc.

Harris, K.S., Robinson, W.J., 1986. The effect of skill level on EMG activity during internal and external imagery. J. Sport Psychol. 8, 105-111.

Hashimoto, R., Rothwell, J.C., 1999. Dynamic changes in corticospinal excitability during motor imagery. Exp. Brain Res. 125, 75-81.

Hecker, J.E., Kazcor, L.M., 1988. Application of imagery theory to sport psychology: some preliminary findings. J. Sport Exerc. Psychol. 10, 363-373.

Herbert, R.D., Dean, C., Gandevia, S.C., 1998. Effects of real and imagined training on voluntary muscle activation during maximal isometric contractions. Acta Physiol. Scand. 163, 361-368.

Holmes, P., Collins, D., 2001. The PETTLEP approach to motor imagery: a functional equivalence model for sport psychologists. J. Appl. Sport Psychol. 13, 60-83.

Hugdahl, K., 1996. Cognitive influences on human autonomic nervous system function. Curr. Opin. Neurobiol. 6, 252-258.

Jackson, P.L., Lafleur, M.F., Malouin, F., Richards, C.L., Doyon, J., 2003. Functional cerebral reorganization following motor sequence learning through mental practice with motor imagery. Neuroimage 20, 1171-1180.

Jacobson, E., 1931. Electrical measurements of neuromuscular states during mental activities. Am. J. Physiol. 96, 115-121.

Jacobson, E., 1932. Electrophysiology of mental activities. Am. J. Psychol. 44, $677-694$.

Jami, L., 1992. Golgi tendon organs in mammalian skeletal muscle: functional properties and central actions. Physiol. Rev. 72, 623-666.

Jeannerod, M., 1994. The representing brain: neural correlates of motor intention and imagery. Behav. Brain Sci. 17, 187-245.

Jeannerod, M., Frak, V., 1999. Mental imaging of motor activity in humans. Curr. Opin. Neurobiol. 9, 735-739.

Jowdy, D.P., Harris, D.V., 1990. Muscular responses during mental imagery as a function of motor skill level. J. Sport Exerc. Psychol. 12, 191-201.

Kleissen, R.F.M., Buurke, J.H., Harlaar, J., Zivold, G., 1998. Electromyography in the biomechanical analysis of human movement and its clinical application. Gait Post 8, 143-158.

Komi, P.V., Linnamo, V., Silventoinen, P., Sillanpää, M., 2000. Force and EMG power spectrum during eccentric and concentric actions. Med. Sci. Sports Exerc. 32, 1757-1762.

Lacourse, M.G., Turner, J.A., Randolph-Orr, E., Chandler, S.L., Cohen, M.J., 2004. Cerebral and cerebellar sensorimotor plasticity following motor imagery-based mental practice of a sequential movement. J. Rehabil. Res. Dev. 41, 505-524.

Lafleur, M.F., Jackson, P.L., Malouin, F., Richards, C.L., Evans, A.C., Doyon, J., 2002. Motor learning procedures parallel dynamic functional changes during the execution and the imagination of sequential foot movements. Neuroimage 16, 142-157.

Li, S., Kamper, D.G., Stevens, J.A., Rymer, W.Z., 2004. The effect of motor imagery on spinal segmental excitability. J. Neurosci. 27, 9674-9680.

Linnamo, V., Strojnik, V., Komi, P.V., 2006. Maximal force during eccentric and isometric actions at different elbow angles. Eur. J. Appl. Physiol. 96, $672-678$.

Lotze, M., Montoya, P., Erb, M., Hulsmann, E., Flor, H., Klose, U., 1999. Activation of cortical and cerebellar motor areas during executed and imagined hand movements: an fMRI study. J. Cogn. Neurosci. 11, 491-501.

Lutz, R.S., 2003. Covert muscle excitation is outflow from the central generation of motor imagery. Behav. Brain Res. 140, 149-163.

McHugh, M.P., Tyler, T.F., Greenberg, S.C., Gleim, G.W., 2002. Differences in activation patterns between eccentric and concentric quadriceps contractions. J. Sports Sci. 20, 83-91. 
Mellah, S., Rispal-Padel, L., Rivière, G., 1990. Changes in excitability of motor units during preparation for movement. Exp. Brain Res. 82, 178-186.

Moritani, T., Muramatsu, S., Muro, M., 1988. Activity of motor units during concentric and eccentric contractions. Am. J. Physiol. Med. 66, 338-350.

Mulder, T., Zijlstra, S., Zijlstra, W., Hochstenbach, J., 2004. The role of motor imagery in learning a totally novel movement. Exp. Brain Res. 154, 211-217.

Mulder, T., de Vries, S., Zijlstra, S., 2005. Observation, imagination and execution of an effortful movement: more evidence for a central explanation of motor imagery. Exp. Brain Res. 163, 344-351.

Naito, E., Kochiyama, T., Kitada, R., Nakamura, S., Matsumura, M., Yonekura, Y., Sadato, N., 2002. Internally simulated movement sensations during motor imagery activate cortical areas and the cerebellum. J. Neurosci. 22, 3683-3691.

Neuper, C., Scherer, R., Reiner, M., Pfurtscheller, G., 2005. Imagery of motor actions: differential effects of kinesthetic and visual-motor mode of imagery in single-trial EEG. Cogn. Brain Res. 25, 668-677.

Newsom, J., Knight, P., Balnave, R., 2003. Use of mental imagery to limi strength loss after immobilization. J. Sport Rehabil. 12, 249-258.

Pfurtscheller, G., Neuper, C., 1997. Motor imagery activates primary sensorimotor area in humans. Neurosci. Lett. 239, 65-68.

Plascencia-Alvarez, N.I., Estanol, B., Corona Figueroa, M.V., Mondragon, B., Lopez-Lomeli, M.M., Espinosa-Sierra, L., Ruiz, J., Garcia-Ramos, G., Infante, O., Martinez, M.R., 2002. Effect of muscle contraction on heart rate and skin blood flow. Preliminary results. Arch. Cardiol. Mex. 72, 13-19.

Ranganathan, V.K., Siemionow, V., Liu, J.Z., Sahgal, V., Yue, G.H., 2004. From mental power to muscle power - gaining strength by using mind Neuropsychologia 42, 944-956.

Roure, R., Collet, C., Deschaumes-Molinaro, C., Delhomme, G., Dittmar, A., Vernet-Maury, E., 1999. Imagery quality estimated by autonomic response is correlated to sporting performance enhancement. Physiol. Behav. 66, 63-72.
Schmidt, R.A., Lee, T.D., 1999. Motor control and learning: A behavioral emphasis, 3rd edn. Human Kinetics, Champaign.

Sequeira, H., Ba M'hamed, S., 1999. Pyramidal control of heart rate and arterial pressure in cats. Arch. Ital. Biol. 137, 47-62.

Shaw, W.A., 1938. The distribution of muscular action potentials during imaging. Psychol. Rec. 2, 195-216.

Shaw, W.A., 1940. The relation of muscular action potentials to imagine weight lifting. Arch. Psychol. 35, 5-50.

Sidaway, B., Trzaska, A., 2005. Can mental practice increase ankle dorsiflexor torque? Phys. Ther. 85, 1053-1060.

Slade, J.M., Landers, D.M., Martin, P.E., 2002. Muscular activity during real and imagined movement: a test of inflow explanations. J. Sport Exerc. Psychol. 24, 151-167.

Smith, D., Collins, D., Holmes, P., 2003. Impact and mechanism of mental practice effects on strength. Int. J. Sport Psychol. 1, 293-306.

Tesch, P.A., Dudley, G.A., Duvoisin, M.R., Hather, B.M., Harris, R.T., 1990. Force and EMG signal patterns during repeated bouts of concentric or eccentric muscle actions. Acta Physiol. Scand. 138, 263-271.

Vernet-Maury, E., Robin, O., Dittmar, A., 1995. The ohmic perturbation duration: an original index to quantify electrodermal response. Behav. Brain Res. 67, 103-107.

Washburn, M.F., 1916. Movement and mental imagery: Outlines of a motor theory of the complexer mental processes. Houghton Mifflin, Boston.

Yue, G., Cole, K.J., 1992. Strength increases from the motor program: comparison of training with maximal voluntary and imagined muscle. J. Neurophysiol. 67, 1114-1123.

Zijdewind, J., Toering, S.T., Bessen, B., Van-Der-Laan, O., Diercks, R.L., 2003. Effects of imagery motor training on torque production of ankle plantar flexor muscles. Muscle Nerve 28, 168-173. 